\title{
Shape of Numbers and Calculation Formula of Stirling Numbers
}

\author{
Ji Peng \\ Department of Electronic Information, Nanjing University, Nanjing, China \\ Email: mcfroo@sina.com
}

How to cite this paper: Peng, J. (2020) Shape of Numbers and Calculation Formula of Stirling Numbers. Open Access Library Journal, 7: e6081.

https://doi.org/10.4236/oalib.1106081

Received: January 16, 2020

Accepted: March 20, 2020

Published: March 23, 2020

Copyright $\odot 2020$ by author(s) and Open Access Library Inc.

This work is licensed under the Creative Commons Attribution International License (CC BY 4.0).

http://creativecommons.org/licenses/by/4.0/

\section{(c) (i) Open Acces}

\begin{abstract}
This article investigated the idea of Shape of numbers and introduced new operators. The idea divides all products of $k$ distinct integers in $[1, n-1]$ into $2^{K-1}$ catalogs and derives the calculation formula of every catalog. As a simple deduction, a direct formula of the Stirling numbers of the first kind $S 1(n, n-$ $k$ ) and a simple recursive formula of Stirling numbers of the second kind $S 2(n, n-k)$ are obtained. By analyzing the Shape of numbers, new congruence formulas are obtained.
\end{abstract}

\section{Subject Areas}

Number Theory

\section{Keywords}

Combinatorics, Shape of Numbers, Stirling Numbers, Congruence Formula

\section{Introduction}

In combinatorial mathematics, Stirling numbers refer to two kinds of numbers [1]: the first and the second. The first kind of Stirling number represents the number of $M$ circles arranged by $N$ different elements. The second kind of Stirling number represents the number of schemes to split $N$ different elements into $M$ sets. Many papers have researched the general calculation formula of the first Stirling number $S 1(n, n-k)$ and the second Stirling number $S 2(n, n-k)$, but general results are not obtained.

This article originate from the solution of the sum of all products of $k$ distinct integers in $[1, n-1]$ without using combination counting.

\section{Shape of Numbers}

$C(N, M)$ is binomial coefficient, Integral polynomial can be expressed as 
$f(n)=\sum K_{i} * C\left(n, m_{i}\right)$ [2]. Here $K_{i}$ is the coefficient, $m_{i}$ is the exponent.

\section{Definition 2.1 operator:}

$$
L_{A} C(N, M)=M * C(N, M+1), \quad L_{B} C(N, M)=(M+1) * C(N, M+2)
$$

It is easy to prove that different operators do not satisfy the commutative law, and the same operator can be written in the form of power.

$$
\begin{gathered}
L_{B}\left(L_{A} C(N, M)\right)=L_{B}(M * C(N, M+1))=(M+2) * M * C(N, M+3) \\
L_{A}\left(L_{B} C(N, M)\right)=L_{A}((M+1) * C(N, M+2)) \\
=(M+2) *(M+1) * C(N, M+3) \\
L_{A}^{2} C(N, M)=M *(M+1) * C(N, M+2) \\
L_{B}^{2} C(N, M)=(M+1) *(M+3) * C(N, M+4) \\
L_{A}\left(L_{A}^{2} C(N, M)\right)=M *(M+1) *(M+2) * C(N, M+3)=L_{A}^{2}\left(L_{A} C(N, M)\right) \\
L_{B}\left(L_{B}^{2} C(N, M)\right)=(M+1) *(M+3) *(M+5) * C(N, M+6) \\
=L_{B}^{2}\left(L_{B} C(N, M)\right)
\end{gathered}
$$

Definition 2.2: Let $f(n)=\sum K_{i} * C\left(n, m_{i}\right)$, then $L_{A} f(n)=\sum K_{i}^{*} L_{A} C\left(n, m_{i}\right), L_{B} f(n)=\sum K_{i}^{*} L_{B} C\left(n, m_{i}\right)$.

Easy to prove:

$$
\begin{gathered}
C(N, M+1)=\sum_{n=0}^{N-1} C(n, M) \\
\sum_{n=0}^{N-1} M * C(n, M)=L_{A} C(N, M) \\
\sum_{n=0}^{N-1}(n-M) * C(n, M)=L_{B} C(N, M) \\
\sum_{n=0}^{N-1} n * C(n-1, M)=L_{B} C(N, M) \\
\sum_{n=0}^{N-1} n * f(n)=L_{A} f(n)+L_{B} f(n)
\end{gathered}
$$

Proof:

$$
\text { Let } \begin{aligned}
f(n)=\sum K_{i} * C\left(n, m_{i}\right) \\
\quad \sum_{n=0}^{N-1} n * f(n) \\
=\sum K_{i} * \sum_{n=0}^{N-1} m_{i} * C\left(n, m_{i}\right)+\sum K_{i} * \sum_{n=0}^{N-1}\left(n-m_{i}\right) * C\left(n, m_{i}\right) \\
=L_{A} f(n)+L_{B} f(n)
\end{aligned}
$$

Definition 2.3: An unordered pair of $M$ different positive integers $\left(K_{1}, K_{2}, \cdots, K_{m}\right)$, sort $K_{i}$ from small to large, there are $M-1$ intervals between adjacent numbers. Use $A$ for continuity and $B$ for discontinuity, record as a string of $M-1$ characters (for example: $A A B B$...) to represents a catalog, define collection of all catalogs as Shape of numbers. Use the symobl $P X$ represent a catelog (if $M=1$ then $P X=1)$. The single $\left(K_{1}, K_{2}, \cdots, K_{m}\right)$ is a Item, $K_{1} * K_{2} * \ldots * K_{m}$ is the product of a item, $K_{i}$ is called the factor.

For example:

$(1,2,4)$ have 2 intervals, the number 1 and 2 is continuous, 2 and 4 is discontinuous, then $P X=A B$; 
(200, 213, 600,601) have 3 intervals, 200 and 213 is discontinuous, 600 and 601 is continuous, then $P X=B B A$;

$(1,2,5,6),(2,3,6,7),(20,21,60,61) P X=A B A ;(1,3,5),(1,3,6),(2,4,6)$, (200, 401, 678) $P X=B B$.

Definition 2.4:

$P M=$ Count of numbers in $P X, P A=$ Count of $A$ in $P X, P B=$ Count of $B$ in $P X$

Obviously, $P M=P A+P B+1$

$|P X|=$ Count of items belonging to $P X$

$\operatorname{MIN}(P X)=$ Minimum product of $P X$, for example: $\operatorname{MIN}(A A)=1 * 2 * 3$, $\operatorname{MIN}(A B)=1 * 2 * 4$

$I D X(P X)=2+P A+2 * P B=P M+P B+1$, for example: $I D X(A A)=4$, $\operatorname{IDX}(A B)=5$

$\operatorname{SUM}(N, P X)=$ Sum of all the product of items belonging to $P X$ in $[1, N-1]$, for example: $\operatorname{SUM}(6, A B)=1 * 2 * 4+1 * 2 * 5+2 * 3 * 5$

$E N D(N, P X)=$ Set of items belonging to $\mathrm{PX}$ with the maximum factor $N-$

1

For example: $\operatorname{END}(6, B)=\{(1,5),(2,5),(3,5)\}$

Obviously:

$E N D(I D X, P X)=\{M I N\}$, the minimum factor of $M I N(P X)$ is 1 , the maximum factor of $M I N(P X)$ is $I D X(P X)-1$.

Easy to prove: $\operatorname{SUM}(N, 1)=C(N, 2) ; \operatorname{SUM}(N, A)=1 * 2 * C(N, 3)$; $\operatorname{SUM}(N, B)=1 * 3 * C(N, 4)$

\section{Definition 2.5:}

$P X+A=\operatorname{Attach} A$ at $P X$ tail

$P X+\mathrm{B}=$ Attach $B$ at $P X$ tail

$P X-1=$ Remove the tail of $P X$

$P X-A=P X$ ends with $A$ and remove the tail

$P X-B=P X$ ends with $B$ and remove the tail

For example: $A B+A=A B A, A B+B=A B B, A B A-A=A B B-B=A B$, it is meaningless to $A B A-B, A B B-A$.

Definition 2.6 short form:

$$
\begin{gathered}
I d x=I D X(P X), \quad I d x A=I D X(P X+A), \quad I d x B=I D X(P X+B), \\
M i n=M I N(P X), \quad M i n A=\operatorname{MIN}(P X+A), \quad M i n B=M I N(P X+B)
\end{gathered}
$$

From definition:

$$
\begin{gathered}
\operatorname{Min} A=\operatorname{Min} * \operatorname{Idx} \\
\operatorname{MinB}=\operatorname{Min} *(\operatorname{Id} x+1) \\
\sum \operatorname{END}(N, P X+A)=(N-1) * \sum \operatorname{END}(N-1, P X) \\
\sum \operatorname{END}(N, P X+B)=(N-1) * \operatorname{SUM}(N-2, P X)
\end{gathered}
$$

(2.1) $\operatorname{SUM}(N, P X+A)=L_{A} S U M(N, P X)$,

$\operatorname{SUM}(N, P X+B)=L_{B} S U M(N, P X)$ 


\section{Proof:}

For $P X=1$, direct validation:

$$
\begin{aligned}
& \operatorname{SUM}(N, A)=1 * 2 * C(N, 3)=L_{A} C(N, 2)=L_{A} \operatorname{SUM}(N, 1) ; \\
& \operatorname{SUM}(N, B)=1 * 3 * C(N, 3)=L_{B} C(N, 2)=L_{B} \operatorname{SUM}(N, 1)
\end{aligned}
$$

For general $P X$ :

$$
\begin{aligned}
\operatorname{SUM}(N, P X+B) & =\sum \operatorname{END}(N, P X+B)+\operatorname{SUM}(N-1, P X+B) \\
& =(N-1) * \operatorname{SUM}(N-2, P X)+\operatorname{SUM}(N-1, P X+B) \\
& =\sum_{n=0}^{N-1} n * \operatorname{SUM}(n-1, P X) \\
& =L_{B} \operatorname{SUM}(N, P X)
\end{aligned}
$$

The last step is obtained from (4).

$$
\begin{aligned}
& \operatorname{SUM}(N, P X+A)+\operatorname{SUM}(N, P X+B) \\
& =(N-1) * \operatorname{SUM}(N-1, P X)+\operatorname{SUM}(N-1, P X+A)+\operatorname{SUM}(N-1, P X+B) \\
& =\sum_{n=0}^{N-1} n * \operatorname{SUM}(n, P X) \\
& =L_{A} S U M(N, P X)+L_{B} \operatorname{SUM}(N, P X) \\
& \quad \rightarrow \operatorname{SUM}(N, P X+A)=L_{A} \operatorname{SUM}(N, P X) \\
& \quad \text { (2.2) } \operatorname{SUM}(N, P X)=\operatorname{Min} * C(N, I d x), \\
& \sum E N D(N, P X)=M i n * C(N-1, I d x-1) \\
& \text { Proof: }
\end{aligned}
$$

$$
\begin{aligned}
& P X=A: \operatorname{SUM}(N, A)=1 * 2 * C(N, 3)=\operatorname{MIN}(A) * C(N, I D X(A)) \\
& P X=B: \operatorname{SUM}(N, B)=1 * 3 * C(N, 4)=\operatorname{MIN}(B) * C(N, I D X(B))
\end{aligned}
$$

For general $P X$, From (2.1):

$$
\begin{aligned}
& \operatorname{SUM}(N, P X+A)=L_{A} \operatorname{SUM}(N, P X)=L_{A}\{\operatorname{Min} * C(N, I d x)\} \\
& =\operatorname{Min} * \operatorname{Id} x * C(N, \operatorname{Id} x+1)=\operatorname{MinA} * C(N, \operatorname{Id} x A) \\
& \operatorname{SUM}(N, P X+B)=L_{B} \operatorname{SUM}(N, P X)=L_{B}\{\operatorname{Min} * C(N, I d x)\} \\
& =\operatorname{Min} *(I d x+1) * C(N, I d x+2) \\
& =\operatorname{Min} B * C(N, \operatorname{Idx} B) \\
& \sum \operatorname{END}(N, P X)=\operatorname{Min} * C(N, \operatorname{Id} x)-\operatorname{Min} * C(N-1, \operatorname{Id} x) \\
& =\operatorname{Min} * C(N-1, I d x-1)
\end{aligned}
$$

q.e.d.

This is a series of formulas, for example:

$$
\begin{gathered}
\operatorname{SUM}(6, A A)=1 * 2 * 3+2 * 3 * 4+3 * 4 * 5=1 * 2 * 3 * C(6,4)=90 \\
\operatorname{SUM}(6, A B)=1 * 2 * 4+1 * 2 * 5+2 * 3 * 5=1 * 2 * 4 * C(6,5)=48 \\
\operatorname{SUM}(6, B A)=1 * 3 * 4+1 * 4 * 5+2 * 4 * 5=1 * 3 * 4 * C(6,5)=72 \\
\operatorname{SUM}(7, B B)=1 * 3 * 5+1 * 3 * 6+1 * 4 * 6+2 * 4 * 6=1 * 3 * 5 * C(7,6)=105 \\
\operatorname{SUM}(8, B B)=\operatorname{SUM}(7, B B)+1 *(3+4+5) * 7+2 *(4+5) * 7+3 * 5 * 7 \\
=1 * 3 * 5 * C(8,6)=420
\end{gathered}
$$




$$
\begin{aligned}
\operatorname{SUM}(8, B A B)= & 1 * 3 * 4 * 6+1 *(3 * 4+4 * 5) * 7+2 * 4 * 5 * 7 \\
= & 576=1 * 3 * 4 * 6 * C(8,7) \\
\operatorname{SUM}(9, B A B)= & \operatorname{SUM}(8, B A B)+1 *(3 * 4+4 * 5+5 * 6) * 8 \\
& +2 *(4 * 5+5 * 6) * 8+3 * 5 * 6 * 8 \\
= & 2592=\operatorname{Min} * C(9,7)
\end{aligned}
$$

\section{The Direct Calculation Formula of $S 1(n, n-k)$}

Definition 3.1: $F_{1}(N, M)=S 1(N, N-M)$, specify $F_{1}(N, 0)=1$;

$F_{1}(N, M)=0(N \leq M)$

Already know $F_{1}(N, 0)=C(N, 0) ; \quad F_{1}(N, 1)=C(N, 2) ;$

$F_{1}(N, N-1)=(N-1)$ ! [3]

$F_{1}(N, M)$ is actually the sum of the products of all $M$ different numbers in $[1, n-1]$, from the definition (3.1) $F_{1}(N, M)=\sum M I N(P X) * C(N, I D X(P X))$, the summation traversal all $P X$ of $P M=M$.

There are $2^{M-1}$ items in the expansion, and the exponent is from $M+1$ to $2 M$

$$
\begin{aligned}
& F_{1}(N, 0)=C(N, 0) \\
& F_{1}(N, 1)=1 !^{*} C(N, 2) \\
& F_{1}(N, 2)=2 ! * C(N, 3)+1 * 3 * C(N, 4) \\
& F_{1}(N, 3)=3 ! * C(N, 4)+1 * 3 * 4 * C(N, 5)+1 * 2 * 4 * C(N, 5) \\
& +1 * 3 * 5 * C(N, 6) \\
& F_{1}(N, 4)=4 ! * C(N, 5)+1 * 3 * 4 * 5 * C(N, 6)+1 * 2 * 4 * 5 * C(N, 6) \\
& +1 * 3 * 5 * 6 * C(N, 7)+1 * 2 * 3 * 5 * C(N, 6) \\
& +1 * 3 * 4 * 6 * C(N, 7)+1 * 2 * 4 * 6 * C(N, 7) \\
& +1 * 3 * 5 * 7 * C(N, 8) \\
& F_{1}(N, 5)=5 ! * C(N, 6)+1 * 3 * 4 * 5 * 6 * C(N, 7)+1 * 2 * 4 * 5 * 6 * C(N, 7) \\
& +1 * 3 * 5 * 6 * 7 * C(N, 8)+1 * 2 * 3 * 5 * 6 * C(N, 7) \\
& +1 * 3 * 4 * 6 * 7 * C(N, 8)+1 * 2 * 4 * 6 * 7 * C(N, 8) \\
& +1 * 3 * 5 * 7 * 8 * C(N, 9)+4 ! * 6 * C(N, 7) \\
& +1 * 3 * 4 * 5 * 7 * C(N, 8)+1 * 2 * 4 * 5 * 7 * C(N, 8) \\
& +1 * 3 * 5 * 6 * 8 * C(N, 9)+1 * 2 * 3 * 5 * 7 * C(N, 8) \\
& +1 * 3 * 4 * 6 * 8 * C(N, 9)+1 * 2 * 4 * 6 * 8 * C(N, 9) \\
& +1 * 3 * 5 * 7 * 9 * C(N, 10) \\
& F_{1}(N, 6)=6 ! * C(N, 7)+1 * 3 * 4 * 5 * 6 * 7 * C(N, 8) \\
& +1 * 2 * 4 * 5 * 6 * 7 * C(N, 8)+1 * 3 * 5 * 6 * 7 * 8 * C(N, 9) \\
& +1 * 2 * 3 * 5 * 6 * 7 * C(N, 8)+1 * 3 * 4 * 6 * 7 * 8 * C(N, 9) \\
& +1 * 2 * 4 * 6 * 7 * 8 * C(N, 9)+1 * 3 * 5 * 7 * 8 * 9 * C(N, 10) \\
& +4 \text { !*6*7*C(N,8)+1*3*4*5*7*8*C(N,9)}
\end{aligned}
$$




$$
\begin{aligned}
& +1 * 2 * 4 * 5 * 7 * 8 * C(N, 9)+1 * 3 * 5 * 6 * 8 * 9 * C(N, 10) \\
& +1 * 2 * 3 * 5 * 7 * 8 * C(N, 9)+1 * 3 * 4 * 6 * 8 * 9 * C(N, 10) \\
& +1 * 2 * 4 * 6 * 8 * 9 * C(N, 10)+1 * 3 * 5 * 7 * 9 * 10 * C(N, 11) \\
& +5 ! * 7 * C(N, 8)+1 * 3 * 4 * 5 * 6 * 8 * C(N, 9) \\
& +1 * 2 * 4 * 5 * 6 * 8 * C(N, 9)+1 * 3 * 5 * 6 * 7 * 9 * C(N, 10) \\
& +1 * 2 * 3 * 5 * 6 * 8 * C(N, 9)+1 * 3 * 4 * 6 * 7 * 9 * C(N, 10) \\
& +1 * 2 * 4 * 6 * 7 * 9 * C(N, 10)+1 * 3 * 5 * 7 * 8 * 10 * C(N, 11) \\
& +4 ! 6 * 8 * C(N, 9)+1 * 3 * 4 * 5 * 7 * 9 * C(N, 10) \\
& +1 * 2 * 4 * 5 * 7 * 9 * C(N, 10)+1 * 3 * 5 * 6 * 8 * 10 * C(N, 11) \\
& +1 * 2 * 3 * 5 * 7 * 9 * C(N, 10)+1 * 3 * 4 * 6 * 8 * 10 * C(N, 11) \\
& +1 * 2 * 4 * 6 * 8 * 10 * C(N, 11)+1 * 3 * 5 * 7 * 9 * 11 * C(N, 12)
\end{aligned}
$$

It can be verified by reference [4].

(3.2) $F_{1}(N, M)=L_{A} F_{1}(N, M-1)+L_{B} F_{1}(N, M-1)=\left(L_{A}+L_{B}\right)^{M-1} F_{1}(n, 1)$

Proof:

From the property of $S 1(n, k)$ [5]

$$
\begin{gathered}
\rightarrow \quad F_{1}(N, M)=(N-1) * F_{1}(N-1, M-1)+F_{1}(N-1, M) \\
\rightarrow \quad F_{1}(N, M)=\sum_{n=0}^{N-1} n^{*} F_{1}(N-1, M)
\end{gathered}
$$

Then it can be proved by (5).

\section{q.e.d.}

It can be understood as: Each product of $F_{1}(N, M)=$ (Product of the first $M-1$ factors $) \times($ Factor $M)$.

Assuming the catalog of first $M-1$ factors is $P X$, then

$L_{A}$ means $P X+A$, no new discontinuity is generated;

$L_{B}$ means $P X+B$, new discontinuity is generated.

From (3.2), even if there is no concept of Shape of numbers, we can get the expansion.

Method 1: recursive method

1: list $F_{1}(N, M-1)$, change new exponent $=($ Original exponent $)+1$, new coefficient $=($ Original coefficient $) \times($ Original exponent $)$

that is $L_{A} F_{1}(N, M-1)$.

2: list $F_{1}(N, M-1)$ again, change new exponent $=($ Original exponent $)+2$, new coefficient $=($ Original coefficient $) \times($ Original exponent +1$)$

that is $L_{B} F_{1}(N, M-1)$.

Method 2: direct method

The expansion of $\left(L_{A}+L_{B}\right)^{M-1}$ is similar to $(A+B)^{M-1}$ without commutative. Coefficient changes according to the arrangement of $A$ and $B$, begin from 1 and from left to right:

in case of $A$, the coefficient has a factor +1 , 
in case of $B$, the coefficient has a factor +2 .

Items, coefficient and exponent are all clear.

For example:

$$
\begin{aligned}
&(A+B)^{3}=A A A+(A A B+A B A+B A A)+(A B B+B A B+B B A)+B B B \\
& F_{1}(N, 4)=1 * 2 * 3 * 4 * C(N, 5) \\
&+(1 * 2 * 3 * 5+1 * 2 * 4 * 5+1 * 3 * 4 * 5) * C(N, 6) \\
& \rightarrow \quad+(1 * 2 * 4 * 6+1 * 3 * 4 * 6+1 * 3 * 5 * 6) * C(N, 7) \\
&+1 * 3 * 5 * 7 * C(N, 8)
\end{aligned}
$$

\section{The Recursive Calculation Formula of $S 2(n, n-k)$}

Definition 4.1. $F_{2}(N, M)=S 2(N, N-M)$, specify $F_{2}(N, M)=0(N<M)$ Already know $F_{2}(N, 0)=S 2(N, N)=1 ; \quad F_{2}(N, 1)=S 2(N, N-1)=C(N, 2)$ [6].

When $M>5$, it's difficult to solve it with combination method.

(4.1) $F_{2}(N, M)=\sum_{n=0}^{N-1}(n-M) * F_{2}(n-1, M-1)$

\section{Proof:}

Already know $S 2(N, M)=S 2(N-1, M-1)+M * S 2(N-1, M) \quad$ [7]

$$
\begin{aligned}
F_{2}(N, M) & =S 2(N, N-M) \\
& =(N-M) * S 2(N-1, N-M)+S 2(N-1, N-M-1) \\
& =(N-M) * S 2(N-1,(N-1)-(M-1))+S 2(N-1,(N-1)-M) \\
& =(N-M) * F_{2}(N-1, M-1)+F_{2}(N-1, M)
\end{aligned}
$$

(4.2) $F_{2}(N, M)=L_{A} F_{2}(N, M-1)+L_{B} F_{2}(N, M-1)-M * \sum F_{2}(N, M-1)$

The proof process is the same as similar to (5).

This is the recursive method:

Exponent is from $M+1$ to $2 M$,

Coefficient of exponent $K$ in $F_{2}(N, M)$

$=\left\{\right.$ Coefficient of exponent $K-1$ in $\left.F_{2}(N, M-1)\right\} *(K-M)$

$+\left\{\right.$ Coefficient of exponent $K-2$ in $\left.F_{2}(N, M-1)\right\} *(K-1)$

$$
\begin{gathered}
F_{2}(N, 0)=C(N, 0) \\
F_{2}(N, 1)=C(N, 2) \\
F_{2}(N, 2)=C(N, 3)+3 * C(N, 4) \\
F_{2}(N, 3)=C(N, 4)+10 * C(N, 5)+15 * C(N, 6) \\
F_{2}(N, 4)=C(N, 5)+25 * C(N, 6)+105 * C(N, 7)+105 * C(N, 8) \\
F_{2}(N, 5)=C(N, 6)+56 * C(N, 7)+490 * C(N, 8) \\
+1260 * C(N, 9)+945 * C(N, 10) \\
F_{2}(N, 6)=C(N, 7)+119 * C(N, 8)+1918 * C(N, 9)+9450 * C(N, 10) \\
+17325 * C(N, 11)+10395 * C(N, 12)
\end{gathered}
$$


Among: $119=56 *(8-6)+1 * 7,1918=490 *(9-6)+56 * 8$, $17325=945 *(10-5)+1260 * 10,10395=0+945 * 11$

$$
\begin{aligned}
F_{2}(N, 7)= & C(N, 8)+246 * C(N, 9)+6825 * C(N, 10)+56980 * C(N, 11) \\
& +190575 * C(N, 12)+270270 * C(N, 13)+135135 * C(N, 14)
\end{aligned}
$$

Among: $190575=17325 *(12-7)+9450 * 11$,

$$
\begin{aligned}
& 270270=10395 *(13-7)+17325 * 12,135135=10395 * 13 \\
& F_{2}(N, 8)= C(N, 9)+501 * C(N, 10)+22935 * C(N, 11)+302995 * C(N, 12) \\
&+1636635 * C(N, 13)+4099095 * C(N, 14) \\
&+4729725 * C(N, 15)+2027025 * C(N, 16)
\end{aligned}
$$

Among: $302995=56980 *(12-8)+6825 * 11$, $4729725=135135 *(15-8)+270270 * 14, \quad 2027025=135135 * 15$

It can be verified by reference [7] [8].

\section{Simple Analysis of the Shape of Numbers}

(5.1) Items of $F_{1}(N, M)$ are divided into $2^{M-1}$ categories according to $P X$, and according to $P B$ (from 0 to $M=1$ ), they can be divided into $P B$ families.

Count of per family $\mid\{P X: P B$ is same $\} \mid=C(M-1, P B)$,

$|P X|=C(N-M, P B+1), \quad|E N D(N, P X)|=C(N-M-1, P B)$

\section{Proof:}

If $P B=0$, find $M$ consecutive numbers from 1 to $N-1$ is equivalent to finding 1 number from 1 to $N-M$.

$$
|P X|=N-M=C(N-M, 1)
$$

If $P B=1,\{P X\}$ with the same $P B$ has the same $|P X|$, we can only calculate $\left|B A^{M-2}\right|$.

Items begin with Number $1: 1 \times 3 \ldots, 1 \times 4 \ldots$, equivalent to finding $M-1$ consecutive numbers from 3 to $n-1$,

$\mid$ Items begin with Number $1 \mid=(N-2)-(M-1)=N-M-1$

Items begin with Number $2: 2 \times 4 \ldots, 2 \times 5 \ldots$, equivalent to finding $M-1$ consecutive numbers from 4 to $n-1$,

$\mid$ Items begin with Number $2 \mid=(N-3)-(M-1)=N-M-2$

So $|P X|=\sum_{i=1}^{N-M-1} C(N-M-i, 1)=C(N-M, 2)$.

It can be proved by mathematical induction

$|P X|=\sum_{i=1}^{N-M-P B} C(N-M-i, P B)=C(N-M, P B+1)$

Verification:

$$
\begin{aligned}
& C(M-1,0)+C(M-1,1)+\cdots+C(M-1, M-1)=2^{M-1} \\
& \sum_{P B=0}^{M-1} C(M-1, P B) * C(N-M, P B+1) \\
& =\sum_{P B=0}^{M-1} C(M-1, P B) * C(N-M,(N-M)-(P B+1)) \\
& =\sum_{P B=0}^{M-1} C(M-1, P B) * C(N-M, N-P A) \\
& =\sum_{P B=0}^{M-1} C(M-1, P A) * C(N-M, N-P A) \\
& =C(N-1, M)
\end{aligned}
$$


Among: $C(M-1, P B)=$ Count of $P X$ with the same $P B$, $C(N-M, P B+1)=|P X|, C(N-1, M)=$ Count of items of $F_{1}(N, M)$.

(5.2) $\sum E N D(I d x+1, P X)=\operatorname{Min} * I d x, \operatorname{SUM}(I d x+1, P X)=\operatorname{Min} *(I d x+1)$

This can be directly calculated by (2.2), and can be proved by mathematical induction.

Proof:

$P X=A, \quad \sum E N D(I D X(A)+1, A)=2 * 3=(1 * 2) * 3=\operatorname{MIN}(A) * \operatorname{IDX}(A)$

$P X=B, \quad \sum \operatorname{END}(\operatorname{IDX}(B)+1, B)=1 * 4+2 * 4=(1 * 3) * 4=\operatorname{MIN}(B) * \operatorname{IDX}(B)$

$\sum E N D(I d x+1, P X)=\operatorname{Min} * I d x$. Note that end items have the same maximum factor, form (8) (9)

$$
\begin{aligned}
& \sum E N D(I d x A+1, P X+A) \\
= & \sum E N D(I d x A, P X) * I d x A=\operatorname{Min} * I d x * I d x A=M i n A * I d x A \\
\sum & E N D(I d x B+1, P X+B) \\
= & S U M(I d x+1, P X) * I d x B=\operatorname{Min} *(I d x+1) * I d x B=\operatorname{MinB} * I d x B
\end{aligned}
$$

q.e.d.

Direct calculation:

(5.3) Average of $\operatorname{SUM}(N, P X)=\operatorname{Min}{ }^{*} C(N, P M) / C(I d x, P M)$; Average of $\sum \operatorname{END}(N, P X)=\operatorname{Min} * C(N-1, P M) / C(I d x-1, P M)$

(5.4) $\operatorname{Min} A=\operatorname{SUM}(I d x+1, P X)-\operatorname{Min}, \operatorname{MinB}=\operatorname{SUM}(\operatorname{Id} x+1, P X)$

(5.5) $\operatorname{SUM}(H * I d x-1, P X)=(H-1) * \sum E N D(H * I d x, P X)$

Gauss computing $1+2+\cdots+(N-1)$ use the method of adding the first and last two terms:

$(1+N-1)+(2+N-2)+\cdots=N *(N-1) / 2$, where $N$ is the sum of each group, $(N-1) / 2$ is the count of groups.

This is a similar way: Take $\sum E N D(N, P X)$ as a whole, select $H \times I d x$ items, sum from small to large, where $(H-1)$ is similar to count of groups, $\sum \operatorname{END}\left(H^{*} I d x, P X\right)$ is similar to the sum of a group.

For example:

$$
\begin{aligned}
& 1+2+3+4+5+6+\cdots \\
& =(1+2)+3+4+5+6+\cdots=3 * 1+(3+4+5+6+\ldots) \\
& =(1+2+3+4)+(5+6+\cdots)=5 * 2+(5+6+\cdots) \\
& 1 * 2+2 * 3+3 * 4+4 * 5+5 * 6+\cdots \\
& =(1 * 2+2 * 3+3 * 4)+4 * 5+5 * 6+\cdots \\
& =4 * 5+(4 * 5+5 * 6+\cdots)
\end{aligned}
$$

(5.6) $\operatorname{SUM}(N, P X) \equiv \sum E N D(N, P X) \equiv 0$ MOD Min

(5.7) When $N$ increases, $\operatorname{Sum}(N, P X)$ with the same $P M$ and $P B$ increases proportionally, regardless of $N$

For example:

$$
\begin{aligned}
& \operatorname{SUM}(N, A A B): \operatorname{SUM}(N, A B A): \operatorname{SUM}(N, B A A) \\
& =M I N(A A B): M I N(A B A): M I N(B A A)
\end{aligned}
$$


(5.8) For prime number $P, \operatorname{SUM}(P, P X) \equiv 0 \operatorname{MOD} P^{*} \operatorname{Min},(\operatorname{Id} x<P)$

From number theory we know $F_{1}(P, M) \equiv 0$ MOD $P,(M<P-1)$ [9] [10], this is its promotion.

For example:

$$
\begin{gathered}
F_{1}(5,2)=1 * 2+2 * 3+3 * 4+1 * 3+1 * 4+2 * 4 \equiv 0 \text { MOD } 5 \\
1 * 2+2 * 3+3 * 4 \equiv 0 \text { MOD } 5 * 1 * 2,1 * 3+1 * 4+2 * 4 \equiv 0 \text { MOD } 5 * 1 * 3
\end{gathered}
$$

(5.9) $\{P X\}$ with the same $P M$ and $P B, P B>0, I D X(P X)=P, P>3$, then $\sum M I N(P X) \equiv 0$ MOD $P *(P-1)$

\section{Proof:}

$F_{1}(P, M) \equiv 0$ MOD $P$, all items of the expansion have the factor $C(P, I d x)$.

If $I d x>P$ then $\operatorname{Sum}(P, P X)=0$, if $I d x<P$ then

$P|\operatorname{Sum}(P, P X) \rightarrow P| \sum \operatorname{Sum}(P, P X), I d x=P$.

For example:

$$
\begin{aligned}
& A B B, B A B, B B A \\
& \rightarrow 1 * 2 * 4 * 6+1 * 3 * 4 * 6+1 * 3 * 5 * 6=5 * 6 * 7 \equiv 0 \text { MOD } 7 * 6 \\
& A A A B, A A B A, A B A A, B A A A \\
& \rightarrow 1 * 2 * 3 * 4 * 6+1 * 2 * 3 * 5 * 6+1 * 2 * 4 * 5 * 6+1 * 3 * 4 * 5 * 6 \\
& \quad \equiv 0 \text { MOD } 7 * 6
\end{aligned}
$$

(5.10) For prime number $P, \quad F_{1}(P-1, M) \equiv 1$ MOD $P \quad(0 \leq M<P-2)$

\section{Proof:}

$F_{1}(P-1,0)=1 \equiv 1$ MOD $P$,

$F_{1}(P-1,1)=(P-1)(P-2) / 2 \equiv(-1) *(-2) / 2 \equiv 1$ MOD $P$

From mathematical induction, if $F_{1}(P-1, M-1) \equiv 1$ MOD P,$(0 \leq M<P-2)$

$$
\begin{aligned}
F_{1}(P, M)= & (P-1) F_{1}(P-1, M-1)+F_{1}(P-1, M) \equiv(P-1)+F_{1}(P-1, M) \\
\equiv & -1+F_{1}(P-1, M) \equiv 0 \operatorname{MOD} P \\
& \rightarrow \quad F_{1}(P-1, M) \equiv 1 \operatorname{MOD} P \quad(0 \leq M<P-2)
\end{aligned}
$$

\section{Conclusions}

In this paper, the concept of Shape of numbers and two new operators were introduced, and through simple way, mainly mathematical induction, the calculation formulas of Shape of numbers were obtained. Just as a simple corollary, calculation formulas of $S 1(N, N-K), S 2(N-K)$ are obtained. And we get some properties of Shape of numbers, especially the new congruence relation from Equations (5.8) and (5.9).

\section{Conflicts of Interest}

The authors declare no conflicts of interest regarding the publication of this paper.

\section{References}

[1] Liu, Y. and Liu, X.S. (2006) Combinatorial Mathematics. Peking University Press, Beijing. 
[2] Hua, L.G. (1957) Number Theory Guidance. Science Press, Beijing.

[3] Tuo, N., Gao, L. and Cai, J.X. (2010) Two Formulas of Stirling Number of the First Kind. Journal of Yan' an University (Natural Science Edition), 3, 4-6.

[4] Xu, C.N. (2013) Operator Proof of Recurrence Formula of Stirling Number of the First Kind. Journal of Inner Mongolia University for the Nationalities (Natural Sciences), 4, 384-385.

[5] Bruald, R.A. (2004) Combinatorial Mathematics. 3rd Edition, China Machine Press, Beijing. (S. X. Feng et al., translation)

[6] Zhang, F.L. (2011) A Property of Stirling Number of the Second Kind. Journal of Weinan Teachers College, 12, 14-16.

[7] Wu, Y.S. (2008) A Formula for the Second Kind of Stirling Number $S 2(n, n-6)$. Journal of East China Jiaotong University, No. 4, 97-99.

[8] Li, M.S. (2009) A Formula of Stirling Number $S(n, n-7)$. Journal of Guangdong Polytechnic Normal University, 3, 16-18.

[9] Hardy, G.H. (2010) An Introduction to the Theory of Numbers. 6th Edition, People's Post and Telecommunications Press, Beijing. (F. Zhang, translation)

[10] Pan, C.D. and Pan, C.B. (2013) Elementary Number Theory. 3rd Edition, Peking University Press, Beijing. 